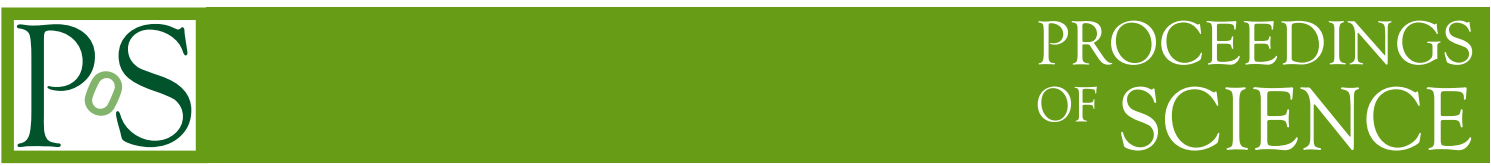

\title{
Muon Radiography with the NOvA Near Detector
}

\section{Peter Filip, for the NOvA Collaboration}

Institute of Physics, Czech Academy of Sciences, Na Slovance 1999/1, Prague, 182 00, Czech Republic

E-mail: filip@fzu.cz

Information on the geological and man-made structures, directly contributing to the overburden of the NOvA Near Detector, located $100 \mathrm{~m}$ underground at Fermilab, are extracted from the cosmic muon data accumulated in the period April 2015 - October 2016. Results of this radiographic analysis of cosmic data are obtained by exploiting the acceptance symmetries of the NOvA detector. Radiographic maps of the overburden variations are acquired without subtracting the surface muon flux (free sky data) and without MC simulation of the cosmic muons attenuation in the homogeneous and flat overburden. By directly subtracting the angular muon track data measured by the NOvA Near Detector, arriving from directions having the same attenuation length (in the flat-surface approximation) and belonging to the acceptance regions with the same reconstruction efficiency, one can efficiently obtain two-dimensional differential radiographic maps.

40th International Conference on High Energy physics - ICHEP2020

July 28 - August 6, 2020

Prague, Czech Republic (virtual meeting) 


\section{Introduction}

NOvA Near Detector is $4 \times 4 \times 16 \mathrm{~m}$ large, reinforced segmented plastic structure [1], containing 157,000 liters of liquid scintillator, equipped with $\sim 19,000$ avalanche photodiodes. Its primary purpose is detection of the elastic and inelastic interactions of $E_{v} \approx 2 \mathrm{GeV}$ neutrinos and antineutrinos, in the selected off-axis angular sector of the NuMI beam, at Fermilab. Cosmic muons steadily penetrate the NOvA Near Detector (100 m underground) at the rate $f_{o} \approx 35 \mathrm{~Hz}$. Charged cosmic tracks and products of the neutrino-induced interactions, occurring in the NOvA detector, are reconstructed using the sophisticated algorithms.
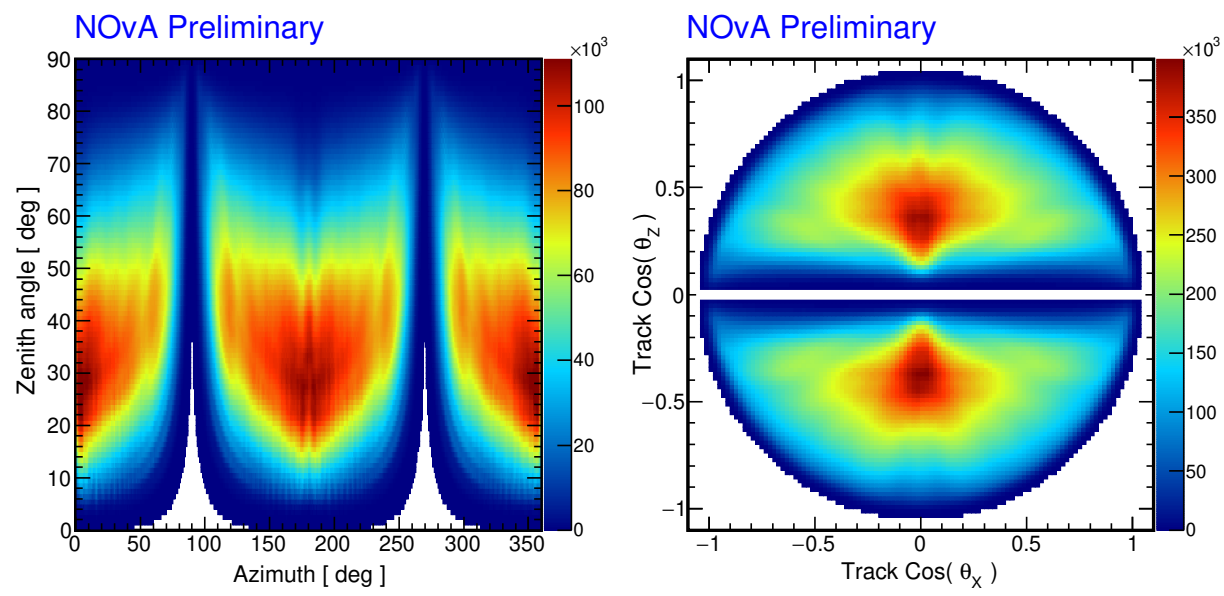

Figure 1: Angular distribution of the cosmic tracks observed in the NOvA Near Detector during the period April 2015 - October 2016. Number of tracks accumulated in the histograms is $1.2 \times 10^{9}$. Smoothing is applied to both histograms. Zero acceptance region is visible at $\left|\cos \left(\theta_{z}\right)\right| \leq 0.02$, and azimuth $90^{\circ}$ and $270^{\circ}$.

Acceptance of the box-shaped NOvA detector is inhomogeneous in azimuth and zenith angle direction. Moreover, DDActivity trigger, accumulating the cosmic data, requires the charged track to cross multiple vertical scintillator planes (each $7 \mathrm{~cm}$ wide). This results in the blind acceptance region for muons arriving from directions orthogonal to the NuMI neutrino beam (see Fig. 1).

However, due to the box-like shape of its fiducial volume, the acceptance of the NOvA detector has discrete left-right and upstream-downstream symmetries, which allows for applying a direct extraction method to obtain differential radiographic maps.

\section{Subtracting the Left-Right and Upstream-Downstream Muon Flux}

Origin of the NOvA coordinate system is at the center of the first detector plane facing the NuMI neutrino beam. $Z$-axis is parallel to the Near Detector longer symmetry axis (NuMI beam direction), $y$-axis is vertically pointing upwards, and $x$-axis is horizontal. Azimuth $\varphi$ and zenith $\theta$ angles of the muon tracks are determined from track angles $\theta_{x}, \theta_{z}, \theta_{y}$ relative to the horizontal $x, z$ and vertical $y$ axis directions. Azimuth $\varphi=0^{\circ}$ is downstream and $\varphi=180^{\circ}$ is upstream direction. $\varphi=0^{\circ}+\phi$ means right, and $\varphi=0^{\circ}-\phi$ means left (by angle $\phi$ ) from the beam direction. Angular distribution of cosmic muon tracks shown in Fig. 1, is upstream-downstream and left-right symmetric, with a small existing deviations (from the perfect symmetry) being barely visible. 
Flat surface and homogeneous overburden above the Near Detector would induce azimuthally symmetric and zenith-angle dependent muon flux attenuation $G(\theta)$ of the surface-level cosmic muon flux $F_{o}(\theta, \varphi) \approx F_{o}(\theta)$, which itself significantly depends on the zenith angle, with a small east-west azimuthal asymmetry (neglected here). Overburden inhomogeneities introduce extra zenith-angle and azimuth-angle dependent attenuation variations, which combined with the detector acceptance give the angular distribution $F(\theta, \varphi)$ of muon tracks in the underground detector:

$$
F(\theta, \varphi)=F_{o}(\theta) \cdot G(\theta) \cdot K(\theta, \varphi) \cdot A(\theta, \varphi)
$$

$K(\theta, \varphi)$ is the angle-dependent "attenuation variations kernel" and $A(\theta, \varphi)$ is detector acceptance, which includes all trigger + analysis + reconstruction efficiencies and detector geometry.
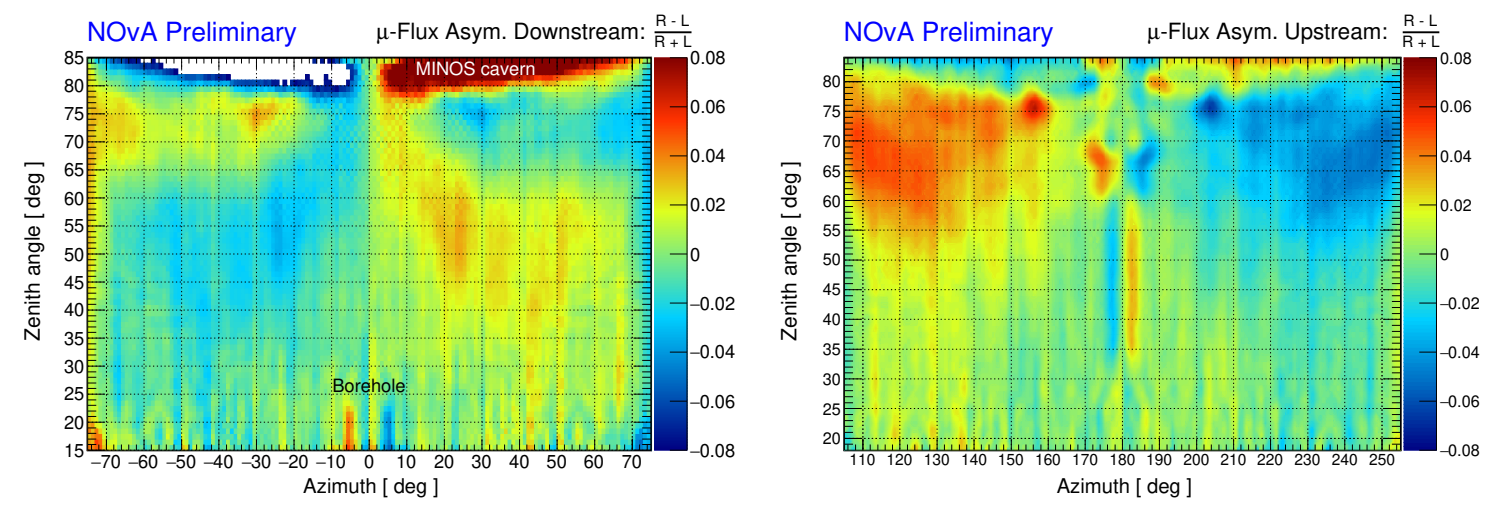

Figure 2: Differential radiographic maps of the upstream and downstream regions above detector, obtained from the cosmic muon tracks observed in the NOvA Near Detector during April 2015 - October 2016 period.

Discrete reflection symmetries of the NOvA detector acceptance: $A\left(\theta, \varphi_{0}+\phi\right)=A\left(\theta, \varphi_{0}-\phi\right)$, existing for $\varphi_{0}=0^{\circ}, 90^{\circ}, 180^{\circ}$, and $270^{\circ}$, allow one to obtain the upstream-downstream $\left(\varphi_{0}=90^{\circ}\right)$ and left-right (upstream: $\varphi_{0}=180^{\circ}$, or downstream: $\varphi_{0}=0^{\circ}$ ) angular differences (asymmetry) of the attenuation variations kernel $K(\theta, \varphi)$, directly from the measured muon flux $F(\theta, \varphi)$, as:

$$
\frac{F\left(\theta, \varphi_{0}+\phi\right)-F\left(\theta, \varphi_{0}-\phi\right)}{F\left(\theta, \varphi_{0}+\phi\right)+F\left(\theta, \varphi_{0}-\phi\right)}=\Delta_{K}\left(\theta, \varphi_{0} \pm \phi\right)=\frac{K\left(\theta, \varphi_{0}+\phi\right)-K\left(\theta, \varphi_{0}-\phi\right)}{K\left(\theta, \varphi_{0}+\phi\right)+K\left(\theta, \varphi_{0}-\phi\right)}
$$

In Fig. 2, we show the resulting asymmetries $\Delta_{K}\left(\theta, 0^{\circ} \pm \phi\right)$ and $\Delta_{K}\left(\theta, 180^{\circ} \pm \phi\right)$ obtained from the cosmic data, using Eq.(2). Pronounced features, visible in Fig. 2, correspond to the man-made and natural overburden (attenuation) variations, present on the surface and within the overburden volume. The MINOS access shaft is visible in the Fig. 2 right panel, at azimuth region $\approx 180^{\circ}$ and $\theta=35^{\circ}-60^{\circ}$ zenith angles. Localized bumps correspond to artificial hills and soil excavations [2].

Symmetric overburden variations will not be visible in differential radiographic maps obtained by the method described here. However, based on the simplicity of the presented approach, one may suggest to apply an adaptation of this method in other cosmic muon radiography projects [3].

\section{References}

[1] D. Ayres, G. Drake et al., The NOvA Technical Design Report 2007-01, DOI:10.2172/935497.

[2] https://www.google.com/maps/@41.839262,-88.269702,18z.

[3] K. Morishima, M. Kuno, A. Nishio et al., Nature 552 (2017) 386-390. 